\title{
Adsorption of Acid Orange II and Cu(II) Ion with Amino-EDTA Modified Silica Gel
}

\author{
Hanyi CHEN $N^{1, a}$, Zhijun XU ${ }^{2,3, b}$, Yunfeng HUANG ${ }^{3, c}$, Zhichao LIN $^{3, d}$, Yanbo \\ ZHANG $^{2,3, \mathrm{e},{ }^{*}}$, Qiang WANG ${ }^{2, \mathrm{f}}$ \\ ${ }^{1}$ South China Institute of Environmental Sciences, Ministry of Environmental Protection of the \\ People's Republic of China, Guangzhou, 510655, China \\ ${ }^{2}$ Engineering Research Center for Clean Production of Textile Dyeing and Printing, Ministry of \\ Education, Wuhan Textile University, Wuhan 430073, China \\ ${ }^{3}$ School of Chemistry and Chemical Engineering, Wuhan Textile University, Wuhan 430073, China \\ ${ }^{*}$ Corresponding author \\ aemail: chenhanyi@scies.org, bemail: xzjwtu@163.com, ${ }^{\mathrm{c} e m a i l: 984701284 @ q q . c o m, ~}$ \\ demail:493687180@qq.com, eemail:Ybzhang_wtu@163.com, ${ }^{f} e m a i l:$ qiang_wang@wtu.edu.cn
}

Keywords: Silica Gel; Adsorption; Copper Ion; Complex; Dye Removal

\begin{abstract}
Silica gel was modified with N-[3-(Trimethoxysilyl)propyl]ethylenediamine, following grafted with ethylenediaminetetraacetic acid (EDTA). The functionalized silica gel was used to readily absorb $\mathrm{Cu}(\mathrm{II})$ ion from aqueous solution forming SG-Cu, via the coordination of EDTA group on the surface of silica gel. The adsorption of Acid Orange II (AO II) in aqueous solution with SG-Cu was investigated under varying time, dye concentration, and adsorbent dosage. The results showed that the adsorption capacity of SG-Cu for AO II increased with increasing of time, initial concentration of the dye solution, and absorbent dosage. The adsorption process followed a pseudo second-order model and experimental data fitted Langmuir adsorption model.
\end{abstract}

\section{Introduction}

Aquatic pollution by synthetic dyes has been a major problem over the past decades because of increased production level and effluent discharge. Variety of industries such as textile, paper and pulp, printing, iron-steel, paints, pharmaceuticals, metal-cleaning, plating and metal-processing have been releasing highly toxic pollutants including dyes and metal ions to the environment [1]. Thus removal of these dyes and metal ions has been the object of great attention in the past few years, not because of toxicity, but also due to unpleasant appearance of polluted surface water [2].

The traditional methods that have been employed for removal of dyes in wastewater are coagulation, ozonation, oxidation, Fenton's reagent, $\mathrm{NaOCl}$, chemical precipitation, coagulation, ion exchange, membrane filtration and evaporation [3, 4]. However, all of the above methods have disadvantages making them less technically appealing in wastewater treatment. Precipitation is ineffective and produces a lot of sludge, chemical oxidation requires extra chemicals and evaporation treatment has high operating costs $[5,6]$. The adsorption process is one of the effective methods for removal dyes and metal ions from the waste effluent. The process of adsorption has an edge over the other methods due to its sludge free clean operation and completely removed dyes, even from the diluted solution $[7,8]$.

As part of our ongoing research of simultaneous removal of metal ions and dyestuffs, in this paper, the applicability of functionalized silica gel derivative as absorbent for copper ions and AO II from water was studied. The effects of variables including the contact time, dye concentration and absorbent dosage were considered.

\section{Experimental}

\section{Materials.}

Silica gel with particle size in the range of 100-200 mesh was purchased from Qingdao Ocean Chemical Company, China. Ethylenediaminetetraacetic acid (EDTA), N, N’-Diisopropylcarbodi- 
imide (DIC) and N-[3-(Trimethoxysilyl) propyl] ethylenediamine (95\%) were obtained from Aladdin Reagent Co. Ltd, China. N, N-Dimethylformamide (DMF), Cupric sulfate $\left(\mathrm{CuSO}_{4} \cdot 5 \mathrm{H}_{2} \mathrm{O}\right)$, Toluene, Methanol, Ether were purchased from Sinopharm Chemical Reagents Co. Ltd, China. These chemicals and reagents were used as supplied without further purification. Dry toluene was distilled under $\mathrm{N}_{2}$ from $\mathrm{CaH}_{2}$ and dry methanol from magnesium methoxide. All aqueous solutions for measurement experiments were prepared by using doubly distilled water, and the other reagents were of analytical grade (AR).

Preparation of functionalized silica gel and its adsorption for copper(II) ions.

Silica gel was pretreated [9] and modified with N-[3-(Trimethoxysilyl)propyl]ethylenediamine, following grafted with ethylenediaminetetraacetic acid (EDTA). The amino-EDTA functionalized silica gel was mixed with $\mathrm{CuSO}_{4}$ solution $\left(1.0 \mathrm{~g}\right.$ of $\mathrm{SiO}_{2}-\mathrm{NH}-\mathrm{NH}-\mathrm{EDTA}$ in $20 \mathrm{~mL}$ of $1.82 \mathrm{mmol}$ $\mathrm{CuSO}_{4}$ ). The yellowish silica gel turned to green-blue shortly, suggesting the fast adsorption of $\mathrm{Cu}(\mathrm{II})$ ion onto the surface of silica gel forming copper (II) bound silica gel, SG-Cu. Above processes are described in Figure 1.

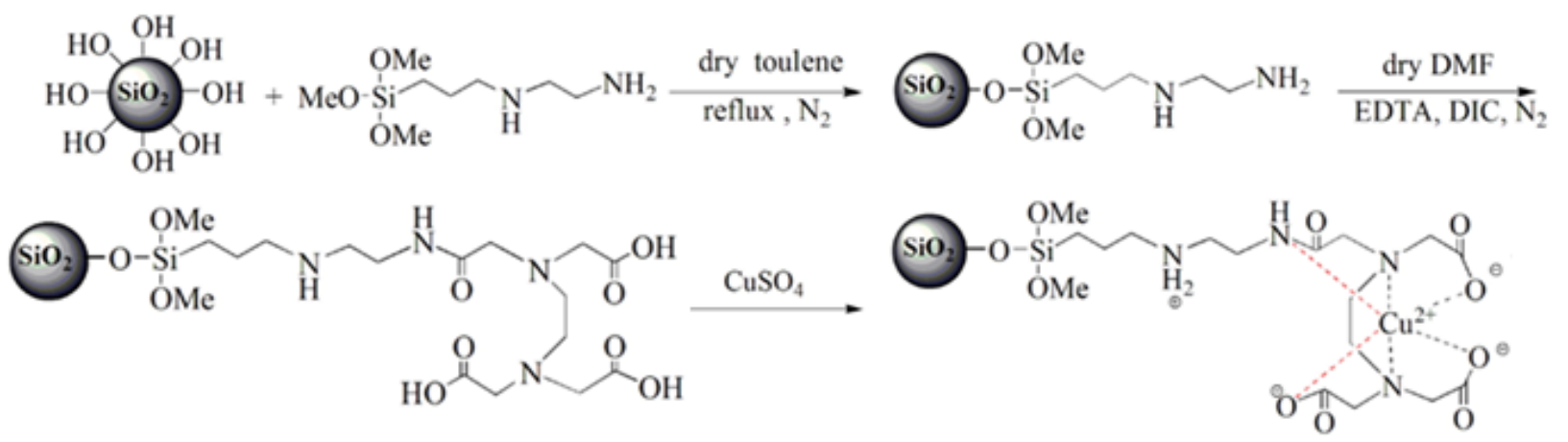

Fig.1. Preparation of functionalized silica gel and its adsorption of copper ions

\section{Adsorption of Acid Orange II.}

The SG-Cu was added in the Acid Orange II (AO II) solution, and the mixture was shaken at room temperature for $3 \mathrm{hrs}$. The dye adsorption percentages were calculated based on the absorbance $\left(\lambda_{\max }=484 \mathrm{~nm}\right)$ of the residue dye solution. The mechanism of the adsorption of anionic dye AOII is depicted in Figure 2.

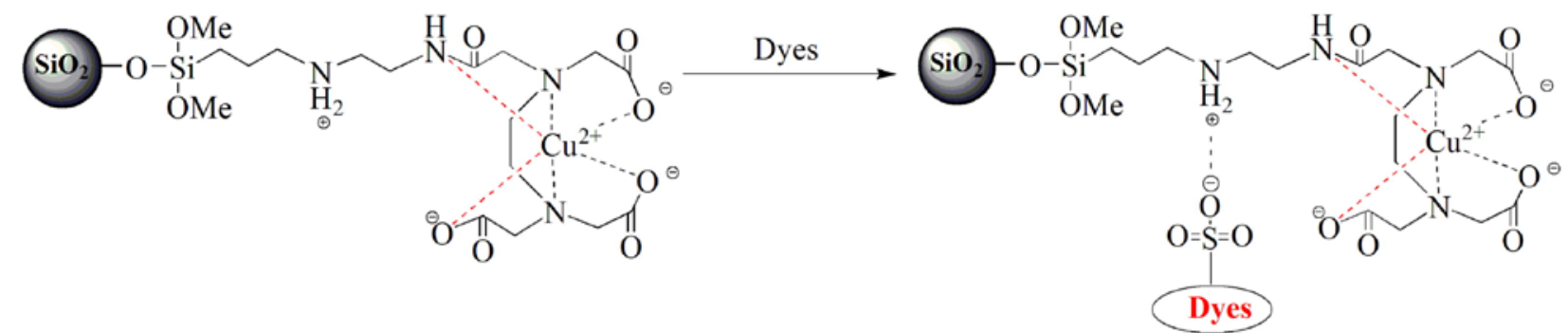

Fig. 2. Mechanism of dyestuff adsorption onto SG-Cu

\section{Results and Discussion}

\section{Effects of contact time.}

The effects of contact time on the removal of AO II by the SG-Cu (30 mg of SG-Cu in $30 \mathrm{~mL}$ of $50 \mathrm{mg} \mathrm{L}^{-1}$ of AO II solution) are shown in Figure 3. Initially the AO II uptake $\left(\mathrm{q}_{\mathrm{t}}\right)$ was fast due to the many vacant adsorption sites. For SG-Cu, all the active sites were occupied by the dye molecules within 45 min after which the adsorption rate gradually decreased and became constant at equilibrium. 


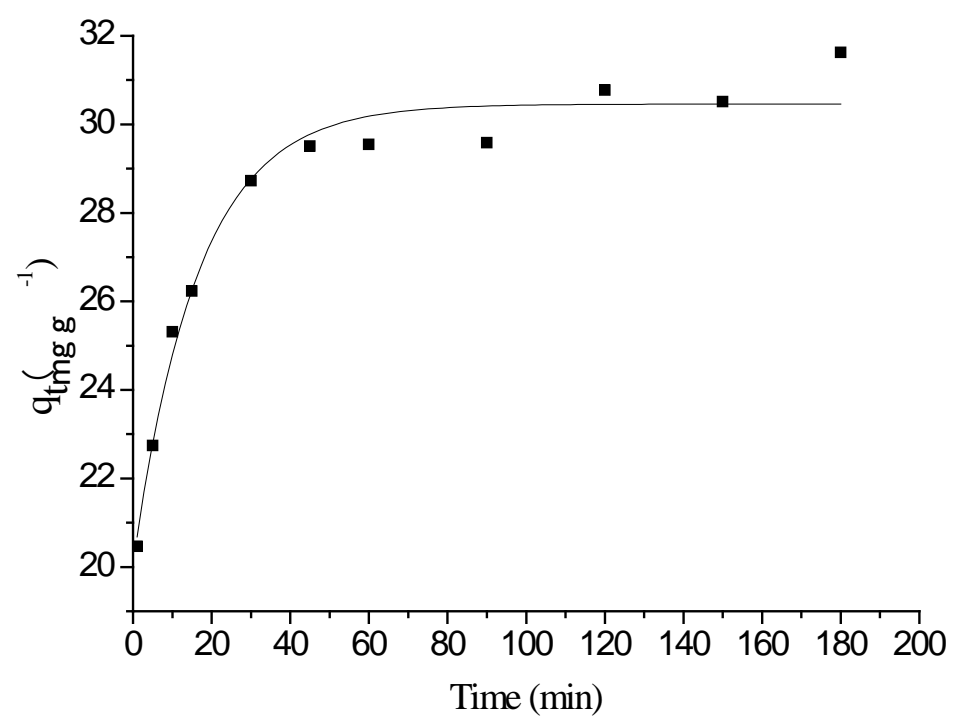

Fig. 3. Effect of contact time on the removal of AO II by SG-Cu

\section{Effects of dye concentration.}

SG-Cu (30 mg) was added to the dye solution $(30 \mathrm{~mL})$ with various dye concentration in the range between 20 to $200 \mathrm{mg} \mathrm{L}^{-1}$, and the effects of dye concentration on removal of AO II by SG-Cu are shown in Figure 4. It indicates that the adsorption capacity $\left(\mathrm{q}_{\mathrm{e}}\right)$ of the SG-Cu increases with increasing of dye concentration, and reaches to maximum value without equilibrium in the concentration range.

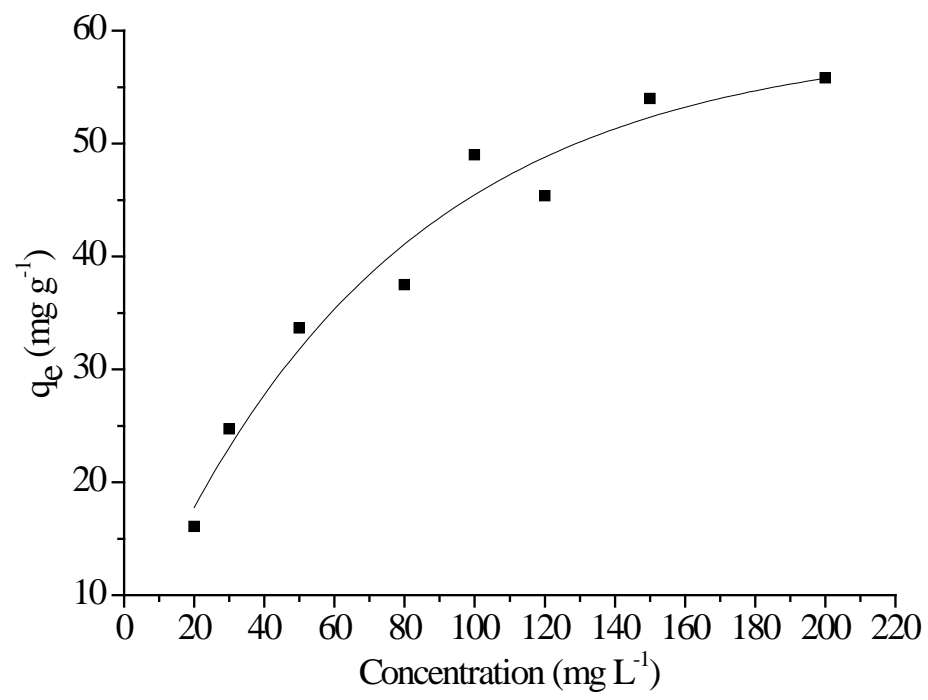

Fig. 4. Effect of dye concentration on the removal of AO II by SG-Cu

\section{Effects of absorbent dosage.}

SG-Cu (20, 30, 40, and $60 \mathrm{mg}$ ) was added into the AO II dye solution (30 mL of $80 \mathrm{mg} \mathrm{L}^{-1}$ ), the effects of absorbent dosage on the removal of AO II are shown in Figure 5. It reveals that the dye removal rate increases when the absorbent dosage increases, since the higher dosage absorbent supply more number of active sites for the interaction with anionic dyes. 


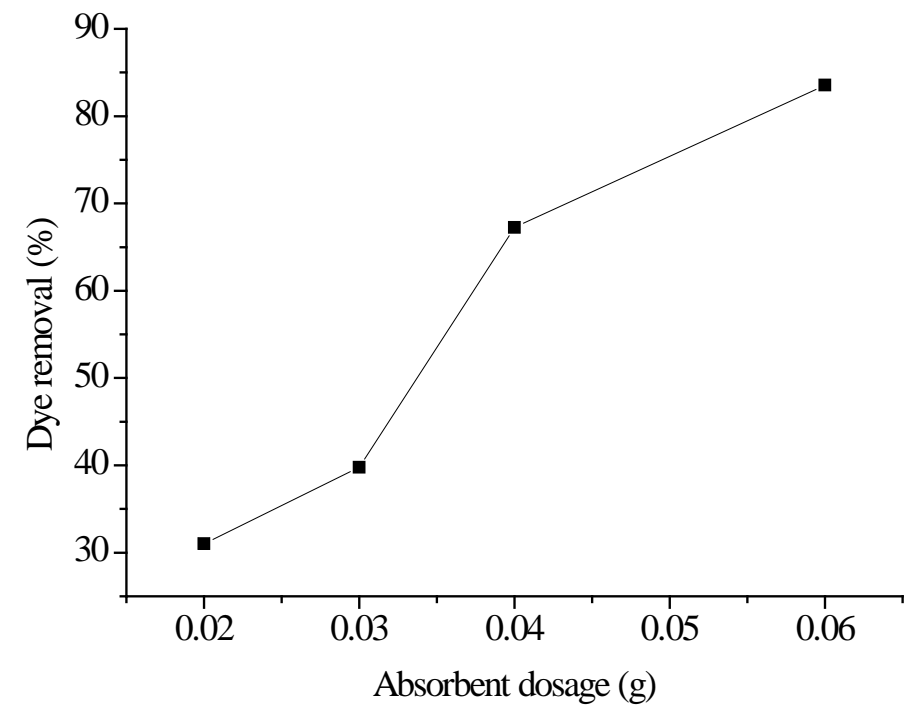

\section{Adsorption kinetics.}

Fig. 5. Effect of absorbent dosage on the removal of AO II by SG-Cu

The pseudo first (1) and second-order (2) models were used to describe the adsorption process of AO II on SG-Cu. The parameters of adsorption kinetics were calculated according to the research [10], and are listed in Table 1. The parameters of $k_{1}$ and $k_{2}$ are the pseudo first-order and second-order rate constant $\left(\mathrm{min}^{-1}\right)$ of adsorption; $\mathrm{q}_{\mathrm{e}}$ (cal.) $\left(\mathrm{mg} \mathrm{g}^{-1}\right)$ values are the amounts of dye adsorbed per unit mass of absorbent at equilibrium. The value of pseudo first-order and second-order rate constant and regression coefficient $\left(\mathrm{R}^{2}\right)$ values are also in Table 1 . The results show that correlation coefficient of pseudo second-order model is better than pseudo first-order model. The calculated qe (qe (Cal.)) based on pseudo second-order model is similar to the experimental data qe (Exp.). Therefore, pseudo second-order model is more effective to represent the adsorption kinetics of SG-Cu for AO II.

$$
\begin{aligned}
& \ln \left(q_{e}-q_{t}\right)=\ln q_{e}-k_{1} t \\
& \frac{t}{q_{t}}=\frac{1}{k_{2} q_{e}^{2}}+\frac{t}{q_{e}}
\end{aligned}
$$

Table 1. Kinetic model parameters for the adsorption of AO II on absorbent

\begin{tabular}{|l|l|l|l|l|l|l|}
\hline \multirow{2}{*}{$\mathrm{q}_{\text {e (Exp.) }}\left(\mathrm{mg} \mathrm{g}^{-1}\right)$} & \multicolumn{3}{|c|}{ Pseudo-first-order } & \multicolumn{3}{c|}{ Pseudo-second-order } \\
\cline { 2 - 7 } & $\mathrm{k}_{1}\left(\mathrm{~min}^{-1}\right)$ & $\mathrm{q}_{\text {e (Cal.) }}\left(\mathrm{mg} \mathrm{g}^{-1}\right)$ & $\mathrm{R}_{1}{ }^{2}$ & $\mathrm{k}_{2}\left(\mathrm{~g} \mathrm{mg}^{-1} \mathrm{~min}^{-1}\right)$ & $\mathrm{q}_{\mathrm{e} \text { (Cal.) }}\left(\mathrm{mg} \mathrm{g}^{-1}\right)$ & $\mathrm{R}_{2}{ }^{2}$ \\
\hline 30.46 & 0.02936 & 6.7017 & 0.7871 & 0.01171 & 31.47 & 0.999 \\
\hline
\end{tabular}

\section{Adsorption thermodynamics.}

Langmuir (3) and Freundlich (4) adsorption isotherms were used to evaluate the adsorption behaviour of SG-Cu for AO II. Table 2 shows $\mathrm{R}_{\mathrm{L}}{ }^{2}$ values of the Langmuir isotherm model for the dye adsorption on SG-Cu is higher than that of the Freundich isotherm model. The results indicate that the adsorption of AO II on SG-Cu fits Langmuir model better than Freundlich model.

$$
\begin{aligned}
& \frac{C_{e}}{q_{e}}=\frac{C_{e}}{q}+\frac{1}{q K_{L}} \\
& \ln q_{e}=\ln K_{F}+\frac{\ln C_{e}}{n}
\end{aligned}
$$

Table 2 Langmuir and Freundlich isotherm parameters

\begin{tabular}{|l|l|l|l|l|l|}
\hline \multicolumn{3}{|c|}{ Langmuir } & \multicolumn{3}{c|}{ Freundlich } \\
\hline $\mathrm{q}\left(\mathrm{mg} \mathrm{g}^{-1}\right)$ & $\mathrm{k}_{\mathrm{L}}\left(\mathrm{L} \mathrm{mg}^{-1}\right)$ & $\mathrm{R}_{\mathrm{L}}{ }^{2}$ & $\mathrm{k}_{\mathrm{F}}\left(\mathrm{mg} \mathrm{g}^{-1}\right)$ & $\mathrm{n}$ & $\mathrm{R}_{\mathrm{F}}{ }^{2}$ \\
\hline 59.3 & 0.0743 & 0.979 & 12.824 & 3.2439 & 0.908 \\
\hline
\end{tabular}




\section{Conclusions}

The amino-EDTA modified silica gel was prepared and it was readily used to fast absorb $\mathrm{Cu}(\mathrm{II})$ ions from aqueous solution, forming $\mathrm{Cu}(\mathrm{II})$ bound silica gel, SG-Cu. SG-Cu were found to effectively adsorb AO II from aqueous solutions. Its adsorption capacity increased with increasing of time, initial concentration of the dye solution, and the SG-Cu dosage. The absorption was fast and reached adsorption equilibrium at about $60 \mathrm{~min}$. The adsorption process of the AO II on SG-Cu followed a pseudo second-order model and experimental data fitted well with Langmuir adsorption model.

\section{References}

[1] Z. Aksu, Application of biosorption for the removal of organic pollutants: A review [J], Process Biochem, 2005, 40:997-1026.

[2] E.T. Burcu, D. Gönül, Single and binary biosorption of heavy metal ions and a reactive dye by aspergillus versicolor [J], Commun. Fac. Sci. Univ. Ank. Series B, 2010, 56:13-25.

[3] T. Robinson, G. Mcmullan, R. Marchant, R. Nigam, Remediation of dyes in textile effluent: A critical review on current treatment technologies with a proposed alternative [J], Bioresource Technol, 2001, 77:247-255.

[4] T.A. Kurniawan, G.Y.S. Chan, W.H. Lo, S. Babel, Physico-chemical treatment techniques for wastewater laden with heavy metals [J], Chem Eng J, 2006, 118:83-98.

[5] B. Volesky, Detoxification of metal-bearing effluents: Biosorption for the next century [J], Hydrometallurgy, 2001, 59 203-216.

[6] T.A. Kurniawan, G.Y.S. Chan, S. Babel, Physico-chemical treatment techniques for wastewater laden with heavy metals [J], Chem. Eng. J., 2006, 118:83-98.

[7] P. Velmurugan, V. Rathina Kumar, G. Dhinakaran, Dye removal from aqueous solution using low cost adsorbent [J], International journal of environmental sciences, 2011, 1:1492-1503.

[8] A. Masoumi, M. Ghaemy, Adsorption of heavy metal ions and azo dyes by crosslinked nanochelating resins based on poly(methylmethacrylate-co-maleic anhydride) [J], eXPRESS Polymer Letters, 2014, 8:187-196.

[9] Q. Wang, W. Gao, Y. Liu, J. Yuan, Z. Xu, Q. Zeng, Y. Li, M. Schröder, Simultaneous adsorption of $\mathrm{cu}(\mathrm{ii})$ and $\mathrm{So}_{4}{ }^{2-}$ ions by a novel silica gel functionalized with a ditopic zwitterionic schiff base ligand [J], Chemical Engineering Journal, 2014, 250:55-65.

[10] Y. Cai, Y. Huang, F. Liu, L. He, L. Lin, Q. Zeng, Liquid ammonia dyeing of cationic ramie yarn with triazinyl reactive dyes [J], Cellulose, 2014, 21:3841-3849. 